\title{
Impact of Patient-Centered Medical Home Implementation on Diabetes Control in the Veterans Health Administration
}

\author{
LeChauncy D. Woodard, MD, MPH', , Omolola E. Adepoju, PhD ${ }^{1,2}$, Amber B. Amspoker, PhD 1,2, \\ Salim S. Virani, $M D, P h D^{1,2}$, David J. Ramsey, $P h D^{1,2}$, Laura A. Petersen, $M D, M P H^{1,2}$, \\ Lindsey A. Jones, $\mathrm{MS}^{7}$, Lea Kiefer, $\mathrm{MPH}^{1,2}$, Praveen Mehta, MD, MBA ${ }^{3}$, and Aanand D. Naik, MD ${ }^{1,2}$ \\ ${ }^{1}$ Center for Innovations in Quality, Effectiveness, and Safety, Michael E. DeBakey VA Medical Center, Houston, TX, USA; ${ }^{2}$ Baylor College of \\ Medicine, Houston, TX, USA; ${ }^{3}$ Veterans Integrated Service Network 12, Westchester, IL, USA.
}

BACKGROUND: Given its widespread dissemination across primary care, the Veterans Health Administration (VA) is an ideal setting to examine the impact of the patient-centered medical home (PCMH) on diabetes outcomes.

OBJECTIVE: To assess the impact of PCMH implementation on diabetes outcomes among patients receiving care in the Veterans Health Administration.

DESIGN: Retrospective cohort analysis and multilevel logistic regression.

PATIENTS: Twenty thousand eight hundred fifty-eight patients in one Midwest VA network who had a diabetes diagnosis in both 2009 and 2012 and who received primary care between October 1, 2008 and September 30, 2009.

MAIN MEASURES: Glycemic and lipid control using VA quality indicators [hemoglobin (Hb) Alc $<9 \%$, low-density lipoprotein cholesterol (LDL-C) $<100 \mathrm{mg} / \mathrm{dL}$ ].

KEY RESULTS: Odds of glycemic control were lower in 2012 than 2009 (OR=0.72, 95\% CI =0.67-0.77, $p<$ $0.001)$, and this change in control over time varied by race (OR of the interaction between time and race $=1.18,95 \%$ $\mathrm{CI}=1.02-1.36, p=0.028$ ). While the disparity in glycemic control between white and black patients persisted post$\mathrm{PCMH}$, the magnitude of the disparity was smaller in 2012 compared to 2009 (2012: $\mathrm{OR}=1.32,95 \% \mathrm{CI}=$ $1.18-1.47, p<0.0001$ and 2009: $\mathrm{OR}=1.59,95 \% \mathrm{CI}=$ $1.39-1.82, p<0.0001)$. Odds of lipid control did not significantly change between 2009 and 2012 and change did not vary by race and/or gender.

CONCLUSIONS: Although there were no significant improvements in odds of lipid control, and odds of glycemic control decreased following PCMH implementation, there was evidence of reduced racial disparities in glycemic control post-PCMH implementation.

KEY WORDS: diabetes; disparities; race and ethnicity; veterans; patientcentered outcomes research.

J Gen Intern Med 33(8): 1276-82 DOI: $10.1007 / \mathrm{s} 11606-018-4386-\mathrm{x}$

(c) Society of General Internal Medicine (outside the USA) 2018

Received August 14, 2017

Revised January 4, 2018

Accepted February 27, 2018

Published online April 2, 2018

\section{INTRODUCTION}

Currently, over two million veterans regularly receive primary care services for diabetes in the Veterans Affairs (VA) healthcare system. ${ }^{1}$ Representing nearly $25 \%$ of all veterans in this system, the VA cares for more than two times the proportion of diabetics in the general population, where diabetes prevalence is estimated at $9 \%$. $^{2}$ To provide more patient-centered, personalized, team-based care, the VA transformed its primary care from the traditional provider centric model to Patient-Aligned Care Teams (PACTs), its model of the Patient-Centered Medical Home (PCMH). PACTs aim to address gaps in chronic disease management through re-organization of primary care delivery to focus on the patient. ${ }^{3}$ With its PACT transformation, the VA, which provides primary care to over five million veterans, became the largest integrated health system to implement the PCMH model. ${ }^{4}$ Aligned with PCMH priorities, VA PACTs aim to deliver team-based care that is comprehensive, patient centered, coordinated, accessible, and data driven. VA PACTs are organized into teamlets consisting of a primary care provider, registered nurse care manager, licensed practical nurse or medical assistant, and clerk who provide care to an assigned panel of approximately 1200 patients. ${ }^{4}$ Teamlets are supported by discipline-specific team members who work with one or more PACTs including pharmacists, social workers, and registered dietitians. ${ }^{5}$

Since its launch in 2010, the PACT model has been implemented nationwide in all VA primary care clinics and has elicited varied responses from providers, ${ }^{4,}{ }^{6-8}$ patients, ${ }^{7}$ and system administrators. Recent evidence on the impacts of PACT highlights significant reductions in potentially preventable events, reduced emergency room and inpatient admissions, improved primary care visits, improved mental health service use, and improved phone and electronic encounters. ${ }^{4}$ ${ }^{7,9}$ Despite these benefits, however, findings regarding quality of care in PCMH models more broadly have been mixed. A review of medical home demonstrations showed generally favorable diabetes outcomes including improved hemoglobin A1c, blood pressure, and lipid control among patients receiving care in a PCMH model. ${ }^{10}$ Conversely, Rosenthal et al. found no differences in diabetes quality indicators between PCMH and usual care groups. ${ }^{11}$ 
In this study, we examine diabetes outcomes for veterans receiving primary care within one Midwestern VA region before and after PCMH implementation. Given its widespread transformation to the PCMH model, the VA serves as an ideal setting to examine the impact of patient-centered, team-based care on diabetes outcomes. We focus on two measures of quality — glycemic control and lipid management — and assess trends pre- and post-implementation of the VA PCMH model. We also assess the impacts of VA PCMH implementation on female and non-Hispanic black patients, two high-risk diabetic populations for whom disparities in care are well documented. ${ }^{12-16}$

\section{METHODS}

\section{Population}

We examined 20,858 diabetic veterans receiving primary care in one Midwestern VA network composed of seven primary care facilities between October 1, 2008, and September 30, 2009. These veterans also received care in 2012, allowing for an examination of diabetes outcomes prior and subsequent to VA PCMH implementation. We identified patients as having diabetes if they had diagnosis codes indicating diabetes (two outpatient or one inpatient code), filled prescriptions for diabetes medications, or at least two outpatient blood glucose readings $>200 \mathrm{mg} / \mathrm{dL} .{ }^{17}$ Consistent with VA quality indicators, ${ }^{18}$ we excluded patients younger than 18 or older than 75 , and those with documented limited life expectancy, including patients receiving hospice care and those with metastatic cancer (see Fig. 1 for inclusion/exclusion criteria).

\section{Patient's Index Date}

We obtained hemoglobin A1c (HbA1c) and low-density lipoprotein cholesterol (LDL-C) laboratory results from the VA administrative databases and the network data warehouse. To determine the patient's $\mathrm{HbA} 1 \mathrm{c}$ and LDL-C index readings, we looked 365 days prior to the patient's last primary care visit. The patient's most recent result was designated as the index date. For patients with no readings, we used their last primary care visit as the index date.

\section{Measures}

Using the VA quality indicators for glycemic control and lipid control, we determined the proportion of patients who achieved index HbA1c levels of $<9 \%$ and LDL-C levels of $<100 \mathrm{mg} / \mathrm{dL}$, respectively. These measures served as our dependent variables. Independent variables included gender and race (non-Hispanic white versus non-Hispanic black), as well as patient characteristics (age, illness burden measured using the Diagnostic Cost Group (DCG)® Relative Risk Score (DCG RRS), coexisting cardiovascular disease, coexisting depression, coexisting hypertension, insulin use at index, statin use at index, number of VA primary care visits in the last year), provider type (physician versus non-physician), and facility type (teaching versus non-teaching). The DCG RRS is a ratio of patient predicted cost to average actual cost of the VA population. A score of 1.00 represents the cost of an "average" patient, a DCG RRS of $<1.00$ represents a lower than average cost (and illness burden), and a score of $>1.00$ represents a higher than average cost (and illness burden). ${ }^{19}$ We used diagnosis and procedure codes contained in the VA clinical and administrative data ${ }^{20}$ to identify comorbid conditions within 2 years prior to the patient's index date.

\section{Statistical Analyses}

We calculated the proportion of diabetic patients with glycemic $(\mathrm{HbA} 1 \mathrm{c}<9 \%)$ and lipid control (LDL-C $<100 \mathrm{mg} / \mathrm{dL})$ in 2009 and in 2012. There were seven total facilities, and all analyses accounted for nesting of patients within facilities. We conducted a series of multilevel logistic regression analyses to examine the effects of time, patient, provider, and facility characteristics on the outcomes of glycemic and lipid control and whether the time effects varied depending on race and/or gender. Statin use was excluded from models predicting glycemic control and insulin use from models predicting lipid control.

For each outcome, we conducted a set of bivariate multilevel logistic regression models, followed by three multivariate multilevel logistic regression models. We began by examining the bivariate associations between time and each outcome and also examined associations between each patient, provider, and facility characteristic and outcome.

Three multilevel logistic regression models predicting each outcome were constructed: (1) a main effects model including time, gender, race, and all other patient, provider, and facilitylevel covariates as predictors; (2) a model including two-way interaction terms between gender and time, race and time, and gender and race as well as all predictors from the main effects model; and (3) a model including a three-way interaction between time, gender, and race, as well as all predictors from the previous models. All continuous variables were centered and dichotomous variables contrast coded $(-0.5$ and 0.5$)$ in models 2 and 3 to eliminate non-essential collinearity and facilitate interpretation of effects. ${ }^{21}$ Importantly, a significant main effect of time would indicate a significant change in outcomes from pre- to post-PCMH. Furthermore, a significant two-way interaction between gender and time, or race and time, would indicate that change in outcomes from pre- to post-PCMH varies by gender or race. A significant two-way interaction between gender and race would indicate that gender differences in outcomes vary by race. A significant threeway interaction between time, gender, and race would indicate that change in outcomes from pre- to post-PCMH varies by gender and race. We followed up significant interactions with simple slope analyses. ${ }^{21}$ We conducted multilevel logistic regression models using the generalized linear models procedure in IBM SPSS Statistics version 23. These models 
Patients in VISN12 facilities who in 2009 were between $18-75$ years of age with diabetes mellitus, who had an assigned PCP, A1c/lipid levels, relevant patient identifiers, and no indication of a limited life expectancy

$(N=34,754$ patients)

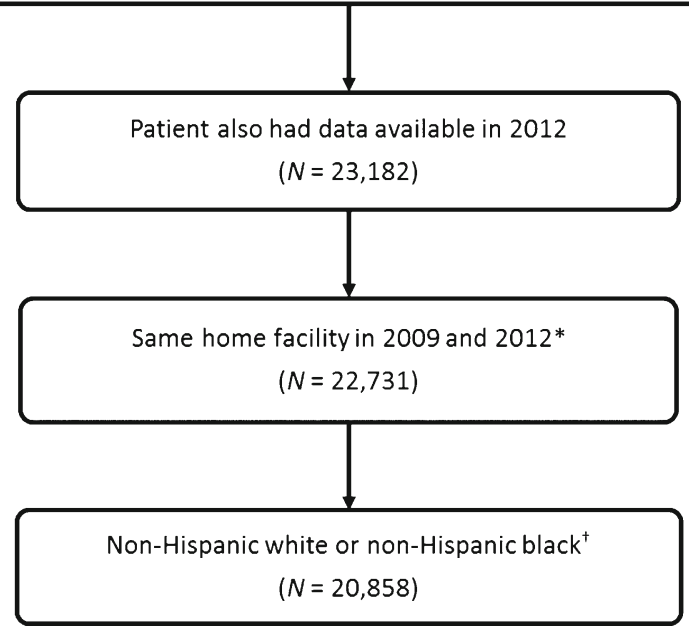

Figure 1 Study inclusion and exclusion criteria. *Participants were nested within facilities, therefore requiring consistent facilities. ${ }^{\dagger}$ Excluded patients with unknown race/ethnicity as well as racial/ethnic groups with very low frequencies.

included the intercept as a random effect and accounted for nesting of patients within facilities. This research was approved by the Baylor College of Medicine Institutional Review Board.

\section{RESULTS}

We included 20,858 patients with diabetes in 2009 and 2012. Table 1 describes the patient, provider, and facility characteristics. Most patients were male $(97.7 \%)$ and non-Hispanic white $(78.4 \%)$. Hypertension $(85.4 \%)$ and statin use $(66.9 \%)$ were prevalent. In $2009,88.1 \%$ of the sample had HbAlc levels $<9 \%$ ( $88.1 \%$ of the males, $85.1 \%$ of the females; $89.8 \%$ of the non-Hispanic whites, $81.8 \%$ of the non-Hispanic blacks). In 2012, glycemic control declined to $85.0 \%$ ( $85.1 \%$ of the males, $80.9 \%$ of the females; $86.5 \%$ of the non-Hispanic whites, $79.7 \%$ of the non-Hispanic blacks). In 2009, 76.2\% of the sample had controlled LDL-C levels of $<100 \mathrm{mg} / \mathrm{dL}$ (76.5\% of the males, $65.5 \%$ of the females; $78.2 \%$ of the non-Hispanic whites, $69.0 \%$ of the non-Hispanic blacks), whereas in 2012, 78.1\% had controlled LCL-C levels (78.3\% of the males, $67.6 \%$ of the females; $79.6 \%$ of the non-Hispanic whites, $72.7 \%$ of the non-Hispanic blacks). Results of bivariate and multivariate multilevel logistic regression models examining predictors of (1) glycemic and (2) lipid control are presented in Tables 2 and 3, respectively.

\section{Predictors of Glycemic Control and Change in Control Pre- to Post-PCMH Implementation}

Bivariate multilevel logistic regression models revealed significant associations between glycemic control and time, gender, race, age, body mass index, marital status, illness burden, depression, hypertension, insulin use, number of primary care visits in the last year, and facility type. In the multivariate multilevel logistic regression model, gender, body mass index, illness burden, hypertension, and facility type were no longer significant. Conversely, cardiovascular disease was significant. Those who were non-Hispanic white $(\mathrm{OR}=$ $1.40,95 \% \mathrm{CI}=1.28-1.52, p<0.001)$, older $(\mathrm{OR}=1.05,95 \%$ $\mathrm{CI}=1.04-1.05, p<0.001)$, married $(\mathrm{OR}=1.17,95 \% \mathrm{CI}=$ $1.09-1.25, p<0.001)$, or had more primary care visits in the last year $(\mathrm{OR}=1.01,95 \% \mathrm{CI}=1.01-1.02, p=0.003)$ were

Table 1 Patient, Provider, and Facility Characteristics $(N=\mathbf{2 0 , 8 5 8}$, Unless Otherwise Noted)

\begin{tabular}{ll}
\hline \hline & $\begin{array}{l}N(\%), \text { unless } \\
\text { otherwise noted }\end{array}$ \\
\hline Characteristic, in 2009 & \\
Non-Hispanic white & $16,355(78.4)$ \\
Male & $20,382(97.7)$ \\
Age (in years), mean (SD) & $61.5(7.2)$ \\
Married & $11,833(56.8)$ \\
Body mass index, mean (SD) & $33.6(6.7)$ \\
Illness burden (RRS), mean (SD) & $1.6(2.2)$ \\
Cardiovascular disease & $6524(31.3)$ \\
Depression & $4194(20.1)$ \\
Hypertension & $17,812(85.4)$ \\
Insulin use & $5339(25.6)$ \\
Glycemic control $(H b A 1 c<9 \%)$ & $18,368(88.1)$ \\
Statin use & $13,944(66.9)$ \\
Lipid control $(\mathrm{LDL}-\mathrm{C}<100 \mathrm{mg} / \mathrm{dL})$ & $15,896(76.2)$ \\
Number of primary care visits in the last & $5.2(3.8)$ \\
year, mean (SD) & \\
Provider type $(N=18,892)$ & \\
Physician & \\
Non-physician & $13,612(72.1)$ \\
Facility type $(N=20,492)$ & $5280(27.9)$ \\
Teaching & \\
Non-teaching & $8454(41.3)$ \\
\hline
\end{tabular}


more likely to have glycemic control than their counterparts. Those with cardiovascular disease $(\mathrm{OR}=0.88,95 \% \mathrm{CI}=$ $0.82-0.94, p<0.001)$, depression $(\mathrm{OR}=0.90,95 \% \mathrm{CI}=$ $0.83-0.97, p=0.006)$, or who used insulin (OR $=0.37,95 \%$ $\mathrm{CI}=0.35-0.39, p<0.001)$ were less likely to have glycemic control than their counterparts.

Importantly, odds of glycemic control were lower in 2012 than $2009(\mathrm{OR}=0.72,95 \% \mathrm{CI}=0.67-0.77, p<0.001)$, and this change in control over time did not vary by gender (interaction between time and gender: $\mathrm{OR}=1.34,95 \% \mathrm{CI}=$ $0.87-2.08, p=0.19)$. However, there was a significant interaction between time and race in the model predicting glycemic control (interaction between time and race: $\mathrm{OR}=1.18,95 \%$ $\mathrm{CI}=1.02-1.36, p=0.028)$. Of note, while the disparity in glycemic control between non-Hispanic white and black patients persisted, the magnitude of this difference was smaller in 2012 compared to 2009 (2012: $\beta=0.28, \mathrm{OR}=1.32,95 \%$ $\mathrm{CI}=1.18-1.47, p<0.0001$ and 2009: $\beta=0.46, \mathrm{OR}=1.59$, $95 \% \mathrm{CI}=1.39-1.82, p<0.0001)$. Stated differently, the odds of glycemic control were lower in 2012 than 2009, especially among non-Hispanic whites (non-Hispanic whites: $\beta=-0.35$, $\mathrm{OR}=0.70,95 \% \mathrm{CI}=0.65-0.76, p<0.0001$ and nonHispanic blacks: $\beta=-0.25$, OR $=0.78,95 \% \mathrm{CI}=0.69-0.88$, $p<0.0001)$. The three-way interaction between time, gender, and race was not a significant predictor of glycemic control $(\mathrm{OR}=0.95,95 \% \mathrm{CI}=0.39-2.34 p=0.91)$.

\section{Predictors of Lipid Control and Change in Control Pre- to Post-PCMH Implementation}

Bivariate multilevel logistic regression models revealed significant associations between lipid control and time, gender, race, age, body mass index, marital status, illness burden, cardiovascular disease, depression, hypertension, statin use, number of primary care visits in the last year, provider type, and facility type. All associations remained in the multivariate multilevel logistic regression model, with the exception of time. Those who were non-Hispanic white $(\mathrm{OR}=1.26,95 \%$ $\mathrm{CI}=1.17-1.35, p<0.001)$, older $(\mathrm{OR}=1.03,95 \% \mathrm{CI}=1.03-$ $1.04, p<0.001)$, married $(\mathrm{OR}=1.14,95 \% \mathrm{CI}=1.08-1.20, p$ $<0.001)$, and had greater body mass index (OR $=1.01,95 \%$ $\mathrm{CI}=1.00-1.01, p=0.001)$, higher illness burden $(\mathrm{OR}=1.02$, $95 \% \mathrm{CI}=1.01-1.04, p<0.001)$, cardiovascular disease $(\mathrm{OR}=1.22,95 \% \mathrm{CI}=1.14-1.29, p<0.001)$, hypertension $(\mathrm{OR}=1.13,95 \% \mathrm{CI}=1.04-1.22, p=0.004)$, used statins $(\mathrm{OR}=2.59,95 \% \mathrm{CI}=2.46-2.74, p<0.001)$, had more primary care visits in the last year $(\mathrm{OR}=1.02,95 \% \mathrm{CI}=1.01-$ $1.02, p<0.001)$, or had a non-physician provider $(\mathrm{OR}=1.10$, $95 \% \mathrm{CI}=1.04-1.17, p=0.002$ ) were more likely to have lipid control than their counterparts. Conversely, females $(\mathrm{OR}=$ $0.82,95 \% \mathrm{CI}=0.69-0.97, p=0.02)$, those with depression $(\mathrm{OR}=0.90,95 \% \mathrm{CI}=0.84-0.96, p=0.001)$, or those at nonteaching facilities $(\mathrm{OR}=0.90,95 \% \mathrm{CI}=0.85-0.96, p<$ $0.001)$ were less likely to have lipid control than their counterparts.
There was not a significant change in odds of lipid control between 2009 and $2012(\mathrm{OR}=0.98,95 \% \mathrm{CI}=0.94-1.05, p$ $=0.75$ ), and this change did not vary by gender (interaction between time and gender: $\mathrm{OR}=1.28,95 \% \mathrm{CI}=0.92-1.78, p$ $=0.15$ ) or race (interaction between time and race: $\mathrm{OR}=1.06$, 95\% CI $=0.94-1.20, p=0.37$ ). The three-way interaction between time, gender, and race was not a significant predictor of lipid control $(\mathrm{OR}=1.30,95 \% \mathrm{CI}=0.63-2.71 p=0.48)$.

\section{DISCUSSION}

We compared diabetes outcomes among veterans within one Midwestern VA network to determine if odds of glycemic and lipid control varied significantly before and after PCMH implementation. Baseline rates of glycemic and lipid control were high among veterans at 88 and $76 \%$, respectively. However, despite widespread PCMH implementation within the VA, odds of glycemic control declined, and after controlling for patient, provider, and facility characteristics, there were no gains in lipid control post-PCMH.

Prior studies have reported mixed findings on impacts of $\mathrm{PCMH}$ adoption. In their examination of quality and efficiency outcomes following transition to PCMH, Fifield and colleagues reported no significant changes in glycemic or lipid control over 2 years. ${ }^{22}$ Friedberg et al. reported no significant differences in glycemic and lipid control among patients in PCMH versus non-PCMH practices over 3 years. ${ }^{23}$ A VA PACT study reported improved clinical quality, reduced inpatient admissions for ambulatory care-sensitive conditions, and reduced emergency department use-only correlated with practices in the top decline of PACT implementation. ${ }^{7}$ Furthermore, an evaluation of the National PCMH Demonstration Project reported small improvements in condition-specific quality of care after 2 years. $^{24}$

We also examined diabetes outcomes pre- and post-PCMH by race and gender. In the past decade, researchers have reported poorer glycemic control among minority veterans compared to non-Hispanic whites, ${ }^{25-27}$ as well as gender disparities in diabetes outcomes among veterans. ${ }^{28-31}$ In our sample, females and non-Hispanic blacks were less likely to have lipid control (relative to males and non-Hispanic whites) and non-Hispanic blacks were less likely to have glycemic control (relative to non-Hispanic whites). However, when we examined gender and race as moderators of the relationship between time and each outcome, we found that racial disparities in glycemic control were reduced in 2012, relative to 2009. Although we observed small differences statistically, our findings are clinically significant in that they provide evidence of the PCMH model's potential to reduce the level of disparity in glycemic control for some groups with historically worse diabetes outcomes.

While racial disparities improved, gender disparities persisted after controlling for patient, provider, and facility characteristics. Our findings are similar to other studies exploring 
gender and racial disparities in diabetes outcomes. ${ }^{26,32-36}$ Tseng et al. (2006) reported no significant gender differences in HbA1c or LDL-C testing among veterans but observed that females were more likely to have uncontrolled lipid levels. ${ }^{35}$ In an analysis of diabetes process of care measures among high-risk patients, lipid control was consistently better among men than women, regardless of race; however, women had greater odds of glycemic control. ${ }^{37} \mathrm{~A}$ cohort study of diabetic adults observed that African American patients in a PCMH were less likely to receive $\mathrm{HbAlc}$ testing and to achieve cholesterol control compared to their white peers. ${ }^{34}$

Though prior studies have documented gender and racial disparities in diabetes care, our study examines such differences within the context of pre- and post-PCMH implementation within the VA. Identified as a key model for addressing racial disparities in chronic diseases, ${ }^{38,39}$ PACT offers the promise of narrowing patient outcome gaps, improving access to care, and fostering veteran-provider relationships. However, our analyses demonstrate limited evidence of PACT effectiveness for reducing disparities among diabetic veterans. These findings are consistent with those of Washington et al. who found ongoing disparities in diabetes outcomes for black, Hispanic and American Indian/Alaska natives receiving VA care. $^{32}$ In addressing gender disparities, work by Yano and colleagues suggests that the PACT model does not include specific elements for gender-focused care. ${ }^{40}$ Others have questioned the availability of the women's health providers and privacy arrangements that make female veterans comfortable and likely to adhere to treatment. Given these findings, PACT implementation efforts need to consider differences in the female veterans' access and use of primary care, their healthcare needs, and building gender-specific workforce competencies. ${ }^{41,}{ }^{42}$ With the burgeoning population increase among female veterans, ${ }^{43}$ the need for gender-specific PACT alignments becomes more consequential. ${ }^{44}$

This study is not without limitations. First, the population of veterans studied is from one geographic region; our results may not be generalizable to the entire veteran population, or to veterans who do not seek care within the VA. Second, our study sample was mostly non-Hispanic white $(77.2 \%)$ and male (97\%), likely reflecting the geography of our data source (a Midwestern VA network). We excluded racial/ethnic groups outside of non-Hispanic black or white, because only very small percentages of diabetic patients receiving care in 2009 and 2012 fell within these categories. The large percentage of male patients in our sample is reflective of the veteran population overall, which is approximately $90 \%$ male. Additionally, our use of a very large dataset allowed for adequate power to make comparisons between males and females and nonHispanic blacks and whites. Third, with a quasi-experimental design, the absence of a control group increases the threat to internal validity. Fourth, we did not consider the degree of PACT transformation - which others have found to be correlated to performance on clinical quality measures. Finally, as our goal was to examine the impact of PACT transformation on diabetes care pre- and post-implementation, our sample is restricted to patients who received VA care at both time points. Despite these limitations, this study contributes significantly to the discourse about impacts of the PCMH on chronic disease management and health equity for vulnerable patient populations.

A major strength of this study is the examination of outcomes among more than 20,000 patients receiving care in the largest integrated health system to implement the PCMH model. ${ }^{4}$ Further, this study includes consideration of racial and gender interactions over time. The positive finding of reduced disparities in glycemic control in 2012 relative to 2009 could be attributed to the PACT focus on cultural preferences and system-wide efforts within the VA to eliminate racial disparities. Continued efforts to adapt the PACT model to address patient care preferences and capacities, like health literacy and activation, may further reduce disparities.

In summary, although there was no evidence of improvement in diabetes care overall, this analysis provides evidence of reduced racial disparities in glycemic control subsequent to PCMH implementation among a large sample of veteran diabetic patients. While non-Hispanic white patients had a $59 \%$ greater odds of glycemic control relative to non-Hispanic black patients pre-PCMH implementation (2009), they had a $32 \%$ greater odds of glycemic control post-PCMH implementation (2012). Future research should identify ways in which PCMH models of care can be improved to better address the needs of chronically ill patients and to determine what factors of the VA PCMH model may have contributed to the reported reduction in racial disparities in glycemic control.

Corresponding Author: LeChauncy D. Woodard, MD, MPH; Center for Innovations in Quality, Effectiveness, and Safety, Michael E. DeBakey VA Medical Center, 2002 Holcombe Blvd., Mail Stop 152, Houston, TX 77030, USA (e-mail: lwoodard@bcm.edu).

Funding Information This work was funded through a contract between VA Network 12 and investigators at the Center for Innovation in Buality, Effectiveness and Safety at the Michael E. DeBakey VA Medical Center. The funding source had no involvement in any aspect of the study design except the initial research question and was not involved in data collection, analysis, or interpretation. We are grateful to the VA Network 12 for their commission and support of this work and to Mark Kuebeler for his statistical support.

\section{Compliance with Ethical Standards:}

This research was approved by the Baylor College of Medicine Institutional Review Board.

Conflict of Interest: The authors declare that they do not have a conflict of interest.

\section{REFERENCES}

1. Wahowiak $\mathbf{L}$. The VA's focus on diabetes care, research, and training. Veterans and Diabetes [Diabetes Forecast web site]. December, 2014: Available at: http://www.diabetesforecast.org/2014/12-dec/veteransand-diabetes.html?referrer=https://outlook.live.com/.

2. Centers for Disease Control Prevention (CDC). National Diabetes Statistics Report: Estimates of Diabetes and Its Burden in the United States, 2014. Atlanta, GA: U.S. Department of Health and Human Services; 2014.

3. Piette JD, Holtz B, Beard AJ, et al. Improving chronic illness care for veterans within the framework of the Patient-Centered Medical Home: 
experiences from the Ann Arbor Patient-Aligned Care Team Laboratory. Transl Behav Med.2011;1:615-23.

4. Rosland AM, Nelson $\mathbf{K}$, Sun $\mathbf{H}$, et al. The patient-centered medical home in the Veterans Health Administration. Am J Manag Care.2013;19:263-72.

5. Petzel RA. Patient Aligned Care Team (PACT) Handbook. Department of Veterans Affairs, Veterans Health Administration. 2014.

6. Jones AL, Cochran SD, Leibowitz A, Wells KB, Kominski G, Mays VM. Usual primary care provider characteristics of a patient-centered medical home and mental health service use. J Gen Intern Med.2015;30:1828-36.

7. Nelson KM, Helfrich C, Sun $\mathbf{H}$, et al. Implementation of the patientcentered medical home in the Veterans Health Administration: associations with patient satisfaction, quality of care, staff burnout, and hospital and emergency department use. JAMA Intern Med.2014;174:1350-8.

8. Sylling PW, Wong ES, Liu C-F, et al. Patient-centered medical home implementation and primary care provider turnover. Med Care.2014;52:1017-22.

9. Kearney LK, Post EP, Pomerantz AS, Zeiss AM. Applying the interprofessional patient aligned care team in the Department of Veterans Affairs: transforming primary care. Am Psychol.2014;69:399-408.

10. Bodjadzievski T, Gabbay R. Patient-centered medical home and diabetes. Med Care.2011;34: 1047-53.

11. Rosenthal MB, Sinaiko AD, Eastman D, Chapman B, Partridge G. Impact of the Rochester Medical Home Initiative on primary care practices, quality, utilization, and costs. Med. Care.2015;53:967-73.

12. Ziemer DC, Kolm P, Weintraub WS, et al. Glucose-independent, blackwhite differences in hemoglobin Alc levels: a cross-sectional analysis of 2 studies. Annals of Intern Med.2010;152:770-7.

13. Herman WH, Dungan KM, Wolffenbuttel BH, et al. Racial and ethnic differences in mean plasma glucose, hemoglobin Alc, and 1,5-anhydroglucitol in over 2000 patients with type 2 diabetes. J Clin Endocrinol Metab.2009;94:1689-94.

14. Herman WH, Ma Y, Uwaifo G, et al. Differences in AlC by race and ethnicity among patients with impaired glucose tolerance in the Diabetes Prevention Program. Diabetes Care.2007;30:2453-57.

15. Selvin E, Parrinello CM, Sacks DB, Coresh J. Trends in prevalence and control of diabetes in the United States, 1988-1994 and 1999-2010. Ann Intern Med.2014;160:517-25.

16. Women Veterans Health Strategic Health Care Group. Gender Differences in Performance Measures, VHA 2008-2011. Washington, DC: Veterans Health Administration; 2012.

17. Woodard LD, Urech T, Landrum CR, Wang D, Petersen LA. Impact of comorbidity type on measures of quality for diabetes care. Med Care.2011;49:605-10

18. Office of Quality and Performance. FY 2011 Q1 Clinical Measures Specification Manual. Vol. 2. Veterans Health Administration; Oct 1. 2010.

19. Petersen LA, Pietz K, Woodard LD, Byrne M. Comparison of the predictive validity of diagnosis-based risk adjusters for clinical outcomes. Med Care.2005;43:61-67.

20. Maynard C, Chapko MK. Data resources in the Department of Veterans Affairs. Diabetes Care.2004; 27:B22-B26.

21. Cohen J, Cohen P, West SG, Aiken LS. Applied Multiple Regression/ Correlation Analysis for the Behavioral Sciences, $3^{\text {rd }}$ ed. Mahwah, NJ: Lawrence Erlbaum Associates.

22. Fifield J, Forrest DD, Burleson JA, Martin-Peele M, Gillespie W. Quality and efficiency in small practices transitioning to patient centered medical homes: a randomized trial. J Gen Intern Med.2013;28:778-86.

23. Friedberg MW, Schneider EC, Rosenthal MB, Volpp KG, Werner RM. Association between participation in a multipayer medical home intervention and changes in quality, utilization, and costs of care. JAMA. 2014;311:815-25.

24. Jaén CR, Ferrer RL, Miller WL, et al. Patient outcomes at 26 months in the patient-centered medical home National Demonstration Project. Ann Fam Med.2010;8:S57-67.
25. Egede LE, Gebregziabher M, Hunt KJ, et al. Regional, geographic, and ethnic differences in medication adherence among adults with type 2 diabetes. Ann Pharmacother.2011;45:169-78.

26. Egede LE, Mueller M, Echols CL, Gebregziabher M. Longitudinal differences in glycemic control by race/ethnicity among veterans with type 2 diabetes. Med Care.2010;48:527-33.

27. Lynch CP, Gebregziabher M, Echols C, Gilbert GE, Zhao Y, Egede LE. Racial disparities in all-cause mortality among veterans with type 2 diabetes. J Gen Intern Med.2010;25:1051-56.

28. Goldstein KM, Melnyk SD, Zullig LL, et al. Heart matters: gender and racial differences cardiovascular disease risk factor control among veterans. Womens Health Issues.2014;24:477-83.

29. Vimalananda VG, Miller DR, Hofer TP, Holleman RG, Klamerus ML, Kerr EA. Accounting for clinical action reduces estimates of gender disparities in lipid management for diabetic veterans. J Gen Intern Med.2013;28:529-35.

30. Vimalananda VG, Miller DR, Palnati M, Christiansen CL, Fincke BG. Gender disparities in lipid-lowering therapy among veterans with diabetes. Womens Health Issues.2011;21:S176-81.

31. Strom Williams JL, Lynch CP, Winchester R, Thomas L, Keith B, Egede LE. Gender differences in composite control of cardiovascular risk factors among patients with type 2 diabetes. Diabetes Technol Ther.2014; 16:421-27.

32. Health Affairs. Racial and Ethnic Disparities Persist at Veterans Health Administration Patient-Centered Medical Homes. Available at: https:// www.healthaffairs.org/doi/full/10.1377/hlthaff.2017.0029. Accessed December 18, 2017.

33. Halanych $\mathbf{J H}$, Wang $\mathbf{F}$, Miller DR, et al. Racial/ethnic differences in diabetes care for older veterans: accounting for dual health system use changes conclusions. Med Care.2006;44:439-45.

34. Simonetti JA, Fine MJ, Chen YF, Simak D, Hess R. Racial comparisons of diabetes care and intermediate outcomes in a patient-centered medical home. Diabetes Care.2014;37:993-1001.

35. Tseng CL, Sambamoorthi U, Rajan M, et al. Are there gender differences in diabetes care among elderly Medicare enrolled veterans? J Gen Intern Med.2006;21:S47-53

36. Tseng CL, Sambamoorthi U, Tiwari A, Rajan M, Findley P, Pogach L. Diabetes care among veteran women with disability. Womens Health Issues.2006;16:361-71.

37. Yu MK, Lyles CR, Bent-Shaw LA, Young BA. Sex disparities in diabetes process of care measures and self-care in high-risk patients. J Diabetes Res.2013;2013:575814.

38. Beal A, Doty MM, Hernandez SE, Shea KK, Davis K. Closing the divide: how medical homes promote equity in health care: The Commonwealth Fund June 2007.

39. Rosenthal TC. The medical home: growing evidence to support a new approach to primary care. J Am Board Fam Med.2008;21:427-40.

40. Yano EM, Haskell S, Hayes P. Delivery of gender-sensitive comprehensive primary care to women veterans: implications for VA patient aligned care teams. J Gen Intern Med.2014;29:S703-7.

41. Lagro-Janssen AL, Canelo I, Yano EM. Creating a roadmap for delivering gender-sensitive comprehensive care for women Veterans: results of a national expert panel. Med Care.2015;53:S156-64.

42. Yano EM, Hayes $\mathbf{P}$, Wright $\mathbf{S}$, et al. Integration of women veterans into VA quality improvement research efforts: what researchers need to know. J Gen Intern Med.2010;25:56-61.

43. US Department of Veterans Affairs. National Center for Veterans Analysis and Statistics. Washington, DC: US Department of Veterans Affairs; Statistics at a Glance (August 2014).

44. Mattocks KM. Care coordination for women veterans: bridging the gap between systems of care. Med Care.2015;53:S8-9. 